\title{
Estudios comparativos del ciclo de vida del mosquito Aedes taeniorhynchus (Diptera: culicidae) de dos colonias de la costa atlántica colombiana
}

\author{
Claribell Hernández ${ }^{1}$, Felio J. Bello ${ }^{2}$, Felisa Durán ${ }^{2}$, Víctor A. Olano ${ }^{1}$, Cristina Ferro ${ }^{1}$
}

\begin{abstract}
Resumen
Este estudio se realizó con el propósito de establecer, en forma preliminar, en el laboratorio, si el mosquito Aedes taeniorhynchus procedente de Barranquilla y Cartagena es realmente una sola cepa y si el proceso de colonización (cría en el laboratorio) influye en el tiempo de duración de las etapas de desarrollo del ciclo de vida de este díptero. Las colonias se establecieron con mosquitos adultos, machos y hembras, recolectados en lugares cercanos a Barranquilla y Cartagena. Los insectos se identificaron taxonómicamente y se colocaron en jaulas Gerberg, en las que se introdujeron tazas con tierra húmeda para que las hembras ovipositaran. De cada localidad, se mantuvieron seis generaciones continuas a una temperatura promedio de $27^{\circ} \mathrm{C}$ y una humedad relativa promedio de $80 \%$. Los estadios inmaduros se alimentaron con Ken-L molido; los adultos de los dos sexos, con solución azucarada y a las hembras se les colocó un curí para que tomaran su comida de sangre. Se registraron los tiempos de duración de cada etapa del ciclo biológico en cada una de las generaciones y se analizaron por medio de la prueba t de comparación de promedios para muestras independientes. Para los tiempos de duración de larva $(\mathrm{t}=1,51)$ y pupa $(\mathrm{t}=0,30)$ de las dos poblaciones del mosquito, no se encontraron diferencias significativas; sin embargo, para el tiempo de eclosión de la larva sí las hubo $(\mathrm{t}=4,21)$. $\mathrm{Al}$ relacionar las seis generaciones de Cartagena no se encontraron diferencias significativas para el tiempo de duración de pupa y eclosión de la larva, pero sí se presentaron a partir de la cuarta generación, para el tiempo de duración de los estadios larvarios. Los resultados de este estudio preliminar sugieren que el mosquito Aedes taeniorhynchus de Barranquilla y Cartagena representa una sola especie. Se demostró, además, que su proceso de colonización, el cual involucra la adaptación de este insecto a las condiciones específicas de un insectario, influye en la duración de su ciclo biológico.
\end{abstract}

\section{Summary}

This pilot study was carried out in the laboratory to establish whether the Aedes taeniorhynchus mosquito, found in Cartagena and Barranquilla, is really a single strain and if the colonisation process (cultured in the laboratory) influences the time taken in the developmental stages of this dipthera's life cycle. Colonies were established with adult mosquitoes, both males and females, collected from sites close to the cities of

${ }^{1}$ Laboratorio de Entomología, INS, Santa Fe de Bogotá, D. C.

${ }^{2}$ Departamento de Química y Biología, Universidad de La Salle, Santa Fe de Bogotá, D. C. 
Cartagena and Barranquilla.The insects were taxonomically identified and placed in Gerberg jars into which cupfuls of humid earth were introduced so that oviposition could take place. Six continuous generations were kept at an average temperature of $27^{\circ} \mathrm{C}$ and $80 \%$ average relative humidity. Immature forms were fed with minced Ken-L; sugar solution was given to adults of both sexes and females were given a guinea pig to provide their blood intake. The duration of each stage of the biological cycle for each generation was recorded and these were analysed by means of the t-test for comparison of averages of independent samples. For larva $(t=1.51)$ and pupa $(t=0.30)$ duration time for the two mosquito populations no significant differences were found; however, hatching time, when it occurred, was $t=4.21$. No significant differences were found for pupa or for larva hatching duration time for the six generations of mosquito from Cartagena, but when significant differences in larval stage duration time were presented, they occurred from the fourth generation onwards. This pilot study's results suggest that the Aedes taeniorhynchus mosquito from Cartagena and Barranquilla represents a sole species. Furthermore, it shows that its colonisation process, being that which involves the adaptation of this insect to the special conditions and have an influence on the duration of its biological cycle.

El mosquito Aedes taeniorhynchus es un eficiente vector del virus de la encefalitis equina venezolana (1). Esta especie presenta una vasta distribución continental, hallándose en las regiones costeras del Atlántico y del Pacífico, incluyendo también las Antillas y las islas Galápagos. En Colombia, se ha encontrado en las costas del Atlántico y del Pacífico y, en el interior del país, en Ambalema, Tolima (2). A pesar de su amplia distribución, no hay estudios que demuestren que se trata de un complejo de especies. En los estudios realizados por Bello et al. (3) y Durán (4) sobre la colonización del mosquito Aedes taeniorhynchus, procedente de dos localidades (Barranquilla y Cartagena), se supone que pertenecen a cepas diferentes. Este estudio pretende, de manera preliminar, establecer si son una sola cepa y si la cría en el laboratorio de este díptero influye en el tiempo de duración de las etapas de desarrollo de su ciclo de vida. Las colonias de Aedes taeniorhynchus establecidas en Estados Unidos y Colombia, entre otras, aunque han proporcinado datos en cuanto al comportamiento del díptero, no hay un informe de estudios comparativos a nivel de laboratorio (5).

\section{Materiales y métodos}

Las formas adultas del mosquito se recolectaron de su hábitat natural, en regiones cercanas a Barranquilla y Cartagena. Los Aedes taeniorhynchus identificados con base en sus características morfológicas externas, se colocaron en el interior de una jaula metálica y se llevaron al laboratorio en donde se adaptaron y mantuvieron bajo condiciones físicas $y$ químicas simuladas, semejantes a las de su hábitat silvestre. Los adultos hembras se alimentaban con sangre de curí. A los adultos machos y hembras se les mantenía permanentemente una bola de algodón envuelta en un pedazo de gasa (chupa) impregnada con una solución azucarada. Para la oviposición, se dejaba en cada jaula un par de tazas plásticas llenas hasta la mitad con tierra húmeda; transcurrido un período aproximado de 9 días, se retiraban de la jaula y se dejaban en incubación por cinco días. Posteriormente, cada taza se colocaba dentro de una cápsula de porcelana que contenía agua en reposo (inundación), donde eclosionaban las larvas. Estas se dejaban en estos recipientes hasta completar su período larvario; cuando se 
presentaba exceso de larvas, se transferían a bandejas esmaltadas. Las larvas se alimentaban una vez por día con Ken-L molido (alimento para perros). Cuando completaban su desarrollo, se iniciaba la fase de pupa. Las pupas se retiraban de las bandejas y se pasaban a tazas con agua que se tapaban hasta que emergían los adultos al día siguiente o a los dos días y se soltaban diariamente al interior de las jaulas.

Para observar el efecto que tiene el proceso de colonización sobre el tiempo de duración del ciclo biológico del mosquito, se utilizó la prueba análisis de varianza de una vía. Para comparar las colonias (Barranquilla-Cartagena) de mosquitos se empleó la prueba $t$ de Student para muestras independientes (5).

\section{Resultados}

Durante el establecimiento y mantenimiento de las colonias (Barranquilla y Cartagena), se obtuvieron seis generaciones y en cada una de ellas se registró el tiempo promedio de duración de los estadios de desarrollo del ciclo de vida del mosquito: huevo, larva, pupa y adulto (macho y hembra) (figura 1). El tiempo promedio de eclosión de las larvas para las colonias de Barranquilla fue de $10,73 \mathrm{~min}$ y para la de Cartagena de 13,9 min.

Las figuras 2 y 3 esquematizan el tiempo promedio de duración del estadio de larvar y de pulpa en las seis generaciones, para las

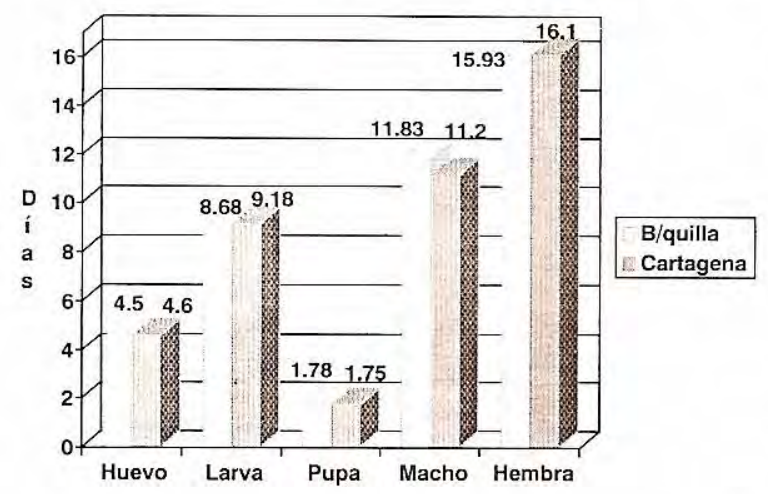

Figura 1. Comparación del tiempo de duración del ciclo de vida de dos colonias de A. taeniorhynchus.

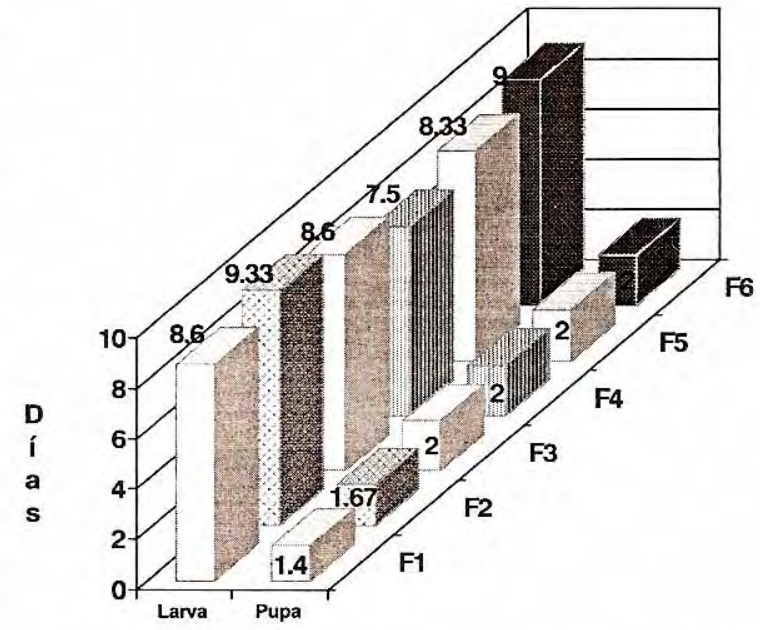

Figura 2. Tiempo de duración de los estadios de larva y de pupa de A. taeniorhynchus (Barranquilla) para seis generaciones.

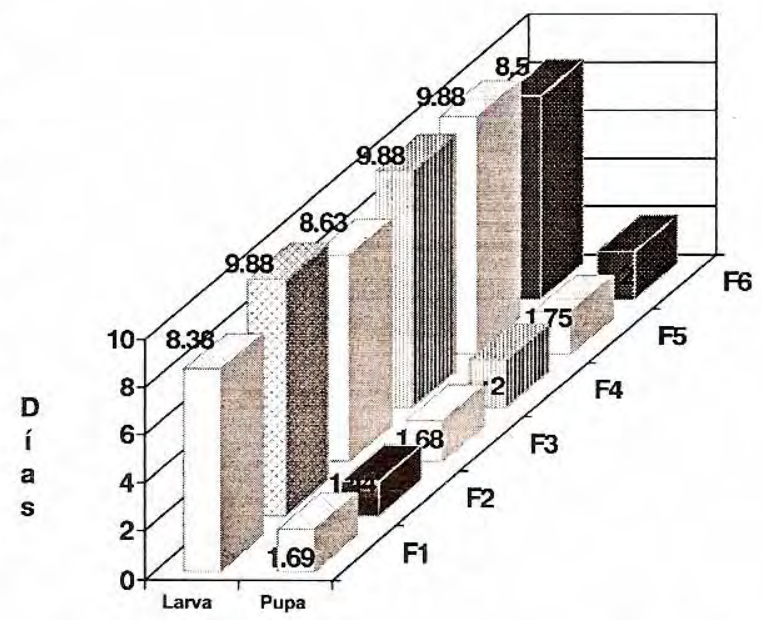

Figura 3. Tiempo de duración de los estadios de larva y de pupa de A. taeniorhynchus (Cartagena) para seis generaciones.

colonas de Barranquilla y Cartagena, respectivamente.

El tiempo de duración de eclosión de las larvas, durante las seis generación en cada una de las colonias, se registra en la figura 4.

\section{Discusión}

Los tiempos de duración del período de larva $(t=1,51)$ y de pupa $(t=0,30)$ de las dos colonias 


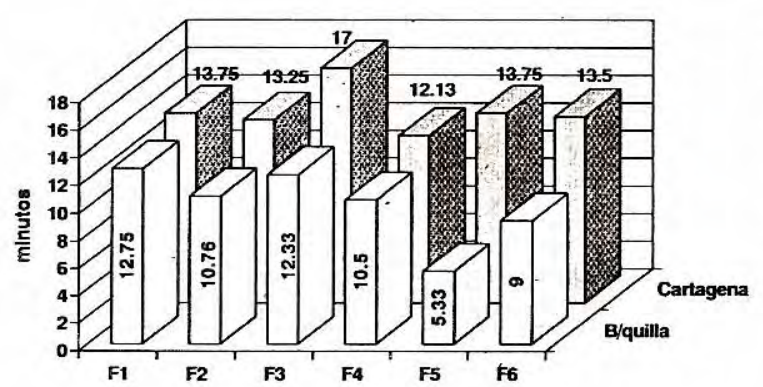

Figura 4. Comparación del tiempo de duración de la eclosión de las larvas en dos poblaciones de $A$. taeniorhynchus para seis generaciones.

del mosquito no presentaron diferencias significativas; sin embargo, en el tiempo de eclosión de la larva $(t=4,21)$ sí las hubo.

Al relacionar las seis generaciones de la colonia de Cartagena no se encontraron diferencias significativas para el tiempo de duración de la pupa y la eclosión de la larva, pero, sí para el tiempo de duración del estadio larvario a partir de la cuarta generación.

Los resultados sugieren que, las colonias de Barranquilla y Cartagena del mosquito Aedes taeniorhynchus, representan una sola especie.
El proceso de colonización de Aedes taeniorhynchus influye en la duración de su ciclo biológico.

Se recomienda hacer un estudio bioquímico de la especie Aedes taeniorhynchus de diferentes localidades con el objeto de establecer si pertenece a un complejo de especies.

\section{Referencias}

1. Sudia WD. Venezuelan equine encephalitis. Washington, D.C.: Pan American Health Organization; 1972.

2. Olano VA. Hallazgo de Aedes taeniorhynchus (Wiedemann, 1921) en un lugar del municipio de Ambalema, departamento del Tolima (Colombia) (Diptera: Culicidae). Biomédica 1985;5:26-8.

3. Bello FJ, et al. Establecimiento y mantenimiento de una colonia de mosquitos Aedes taeniorhynchus (Diptera: Culicidae), cepa Barranquilla. Biomédica 1993;14(2):69-76

4. Durán F. Establecimiento y mantenimiento de una colonia de Aedes taeniorhynchus (Diptera: Culicidae) cepa Cartagena y su estudio cariológico en condiciones in vivo (tesis). Santa $\mathrm{Fe}$ de Bogotá: Universidad de La Salle; 1996.

5. Gerberg EJ. Manual for mosquito rearing and experimental techniques. American Mosquito Control Association Bulletin 1970;5.

6. Sokal RR, Rohlf FJ. Biometría, principios y métodos estadísticos en la investigación biologica. España; 1979;220-3. 